\title{
STATISTICAL ANALYSIS OF THE WORKING PROCESS FOR VERTICAL ROTOR HARROWS
}

\author{
Valentin Iordache ${ }^{1}$, Ion Saracin ${ }^{1}$, Sorin Dan Iordache ${ }^{2}$ \\ ${ }^{1}$ University of Craiova, Romania; ${ }^{2}$ University Politehnica of Bucharest, Romania \\ valentinjordacheyahoocom, ion_saracin@yahoo.com, jordacheeng@yahoo.com
}

\begin{abstract}
The paper presents the results of the statistical analysis on the qualitative indices of the working process of vertical rotor harrows for preparing the germination bed in order to establish agricultural crops: the degree of soil shredding, the degree of soil levelling, as well as energy indices of the work: tensile strength, fuel consumption and fuel consumption specific to the productivity unit. For each of the quality indices of the work theoretical expressions were obtained that show their dependence on some of the main operating parameters of vertical axis rotor harrows: working speed, speed of the harrow rotors and the number of knives that equip each rotor. The purpose of the static analysis is to show which of the quality indices and energy indices of the work process have a real optimization potential for the work process. The results obtained were used in statistical models that can indicate a higher degree of generality. Capitalization of statistical results is for validation and/or correction of theoretical or theoretical-empirical models of technical-economic quality indices: consumption, productivity. From the statistical analysis it resulted that one of the quality parameters (energy) deeply involved in the calculation of consumption is the tensile strength, which theoretical-empirical model was based on the experimental results presented in this article. Statistical models provide relationships between quality parameters of works performed by agricultural machines and input and control (adjustment) parameters of the work process. In this way it becomes possible to optimize the quality, possibly simultaneously with the energy and economic optimization of the work process.
\end{abstract}

Keywords: rotor, knife, harrow, productivity.

\section{Introduction}

In this paper the parameters are presented that characterize the working process of vertical axis rotor harrows, the results obtained experimentally refer to qualitative indices of the working process of vertical rotor harrows for preparation of the germination bed: degree of soil shredding, level, as well as the energy indices of the works: tensile strength, fuel consumption and fuel consumption specific to the productivity unit. Qualitative work indices of germination bed preparation work characterize the degree to which the agrotechnical requirements imposed on the soil are currently met [1;2]. If economic indices can be presented theoretically, the qualitative ones regarding the condition of the cultivated soil are impossible to estimate in the absence of experimental data. Even when estimating economic quality indicators, it is preferable to perform experiments or at least experimentally verify [3;4].

The main objectives of this paper referred to the parameters that characterize the working process for rotary harrows with vertical axis rotors, the relationships between them and the identification of possibilities to optimize the working process using found relationships.

The aggregate studied in this paper already has an appreciable duration of exploitation. Over time, interest in the rotary cutter work has partially decreased, because some solutions are affected beyond the allowable limit in terms of their texture (porosity, [5]).

However, the studies assigned to the rotary harrows are numerous, so that their experience can be used to formulate an elementary mathematical model, useful in order to select the parameters of machine operation before performing the experiments. The problem is to make the experiments as efficient as possible, because they are costly and consuming on the work front, often unavailable in the current conditions. The efficiency of the experiences is materialized in the capacity not to omit important operating parameters, at the same time to establish a hierarchy of important operating parameters, so that some of the least important ones can be neglected.

The results were used for higher statistical models that may have a higher degree of generality. The third way of capitalizing on the results is the validation and/or correction of some theoretical or theoretical-empirical models of the technical-economic indicators of quality: consumption, productivity [5; 6]. An interesting work that appeared exactly in this field, is [7]. It deals with the definition of the critical kinematic parameters of the rotary harrow with the vertical axis of rotation. The kinematic parameter in [7] is defined as the ratio between the pre-peripheral speed of the blade working in the ground and the speed of the machine advancing in the field. We note from [7] the importance of the 
kinematic parameters: the forward speed and peripheral speed of the rotor blade, so both the rotational speed of the rotor and the radius on which the blade is arranged. In addition, [7], it also takes into account the working depth. The author [7] varies the forward speed and working depth and maximizes the crushing percentage of earth clumps.

Also following an experimental study, the authors [8] conclude that the granulation is finer, if the rotation speed of the wheels is higher. In addition, the fuel consumption of the unit increases linearly with the angular speed of the rotors. Therefore, the authors [8] essentially aim to increase the fineness of the soil granules with the rotation speed of the rotor knives, only two parameters and estimate a third, related to the process efficiency, fuel consumption.

Another interesting experimental study, [9], establishes that rotary harrows with vertical and horizontal axis increase the degree of outside of the soil and its density. They consider in their experiments [9] as variables the speed of advance of the aggregate and the depth of work. The forward speed of the unit and the speed of the rotary harrows with vertical axis are the main parameters varied in the paper [10]. The authors [11] take into account in the experimental study of rotary harrows, in addition to the parameters listed above in previous studies, the soil moisture. Experimental studies on rotary harrows are numerous today, due to the many problems that must be solved relative to their operation, [12;13].

\section{Materials and methods}

The materials used in the experimental research consist of: agricultural harrow with rods with vertical axis, which was equipped with two, three and four knives on the rotor in the unit with a tractor of $80 \mathrm{HP}$, Fig.1. The experimental results used are obtained in a reddish-brown forest soil with an average humidity of $16.83 \%$ in the range of $0-5 \mathrm{~cm}$ depth, an average humidity of $21.3 \%$ in the range of $5-10 \mathrm{~cm}$ and an average humidity of $23.73 \%$. in the depth range $10-15 \mathrm{~cm}$. The average temperature in the soil was $21.97^{\circ} \mathrm{C}$. The density of this soil varies, depending on humidity, between 1350 and $1550 \mathrm{~kg} \cdot \mathrm{m}^{-3}$.

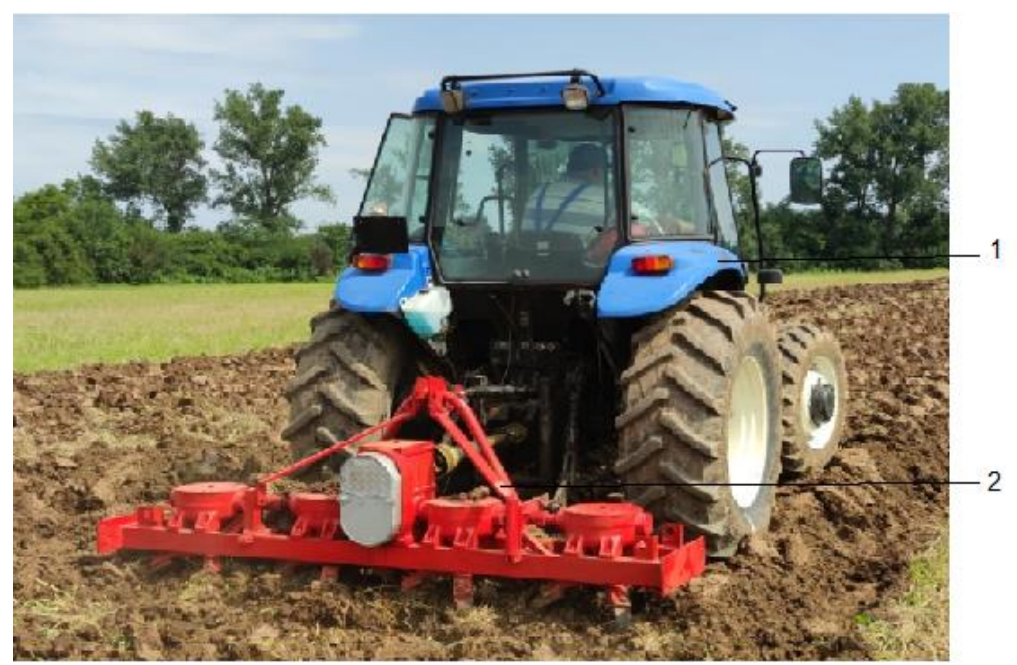

Fig. 1. Agricultural harrow unit with vertical rotor in operation: 1 - tractor; 2 - harrows with vertical rotor

The measuring equipment was used for various types of measurements:

- simple equipment for measuring the leveling level, the degree of crushing, the traction of the tractor, the working speed (Fig. 2, 3);

- apparatus for measuring the soil moisture and penetration resistance ;

- apparatus for tensometric measurements (measuring and data acquisition and recording equipment) and the apparatus for measuring the moment at the socket and for the acquisition and storage of the data taken from it [4]. 


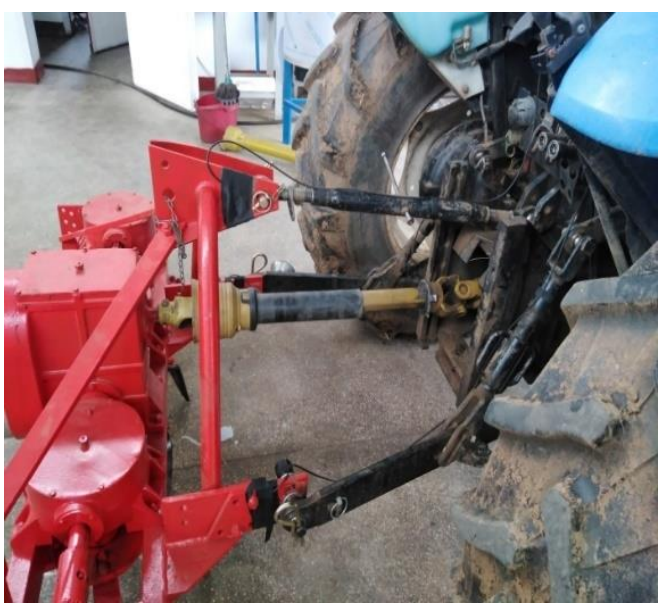

Fig. 2. Tensometric mark glued and protected on $1000 \mathrm{Nm}$

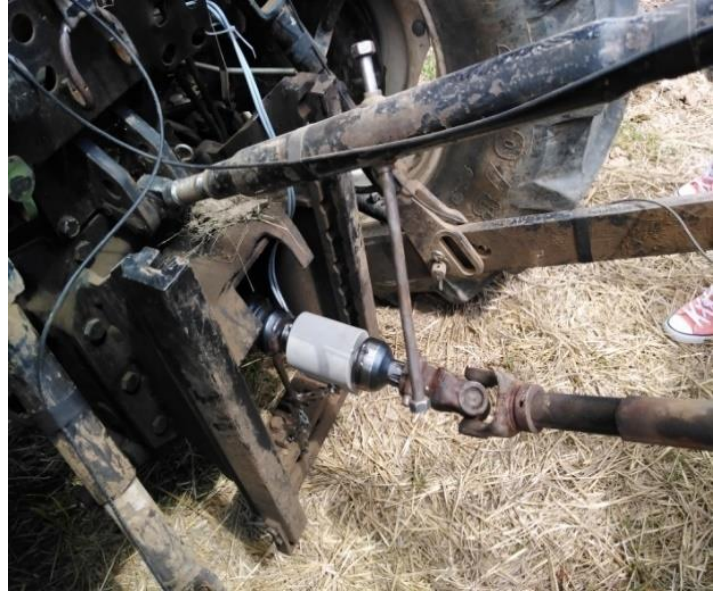

Fig. 3. Tractor torque transducer, HBM 1000 GARV beam coupled to the central tie rod

\section{Results and discussion}

The graphical representations of the sequences of tensometric measurements recorded in the work were used in numerical form to calculate the average tensile strength in each of the control parameters (working speed, rotor speed, number of knives mounted on each rotor). A typical sequence is that of Fig. 4.

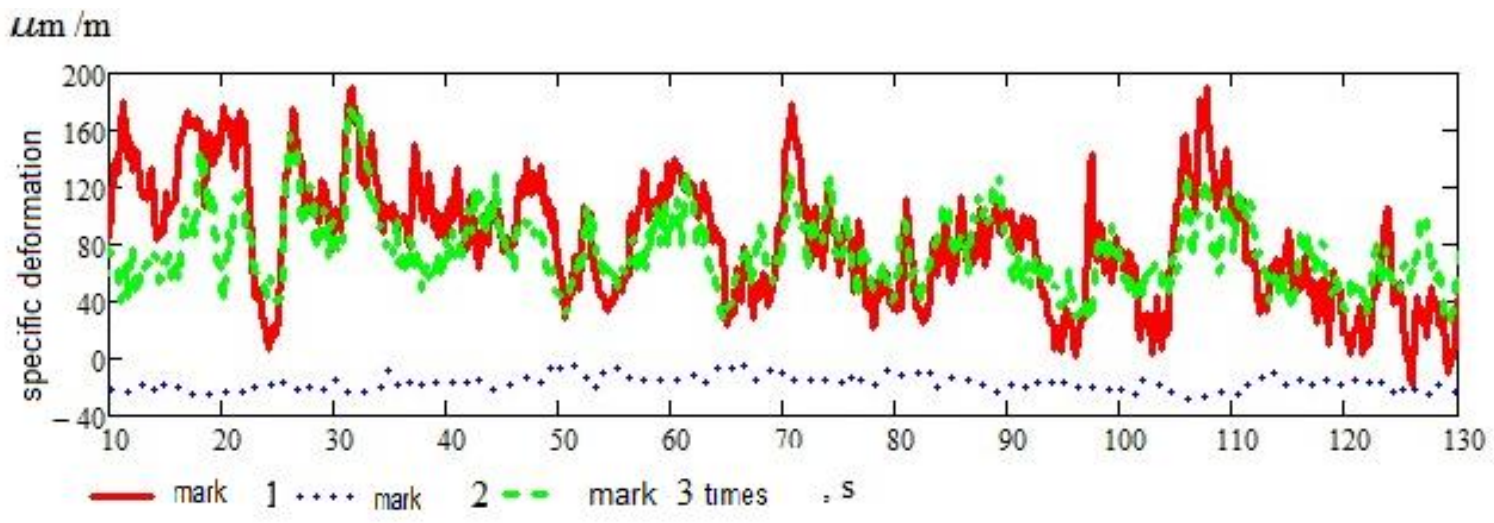

Fig. 4. Tensometric recording sequence obtained during the experiment performed with working speed $0.39 \mathrm{~km} \cdot \mathrm{h}^{-1}$, rotor speed $173 \mathrm{rpm}$ and $3 \mathrm{knives}$ mounted on each rotor

Degree of soil leveling. One of the important parameters of the quality of the work, the distribution of the measurements in the field on the initial and the processed surface is shown graphically in Fig. 4, and 5. These graphical representations facilitate the visibility of the effect of mechanical soil processing made by the harrow with rotors with vertical axis.
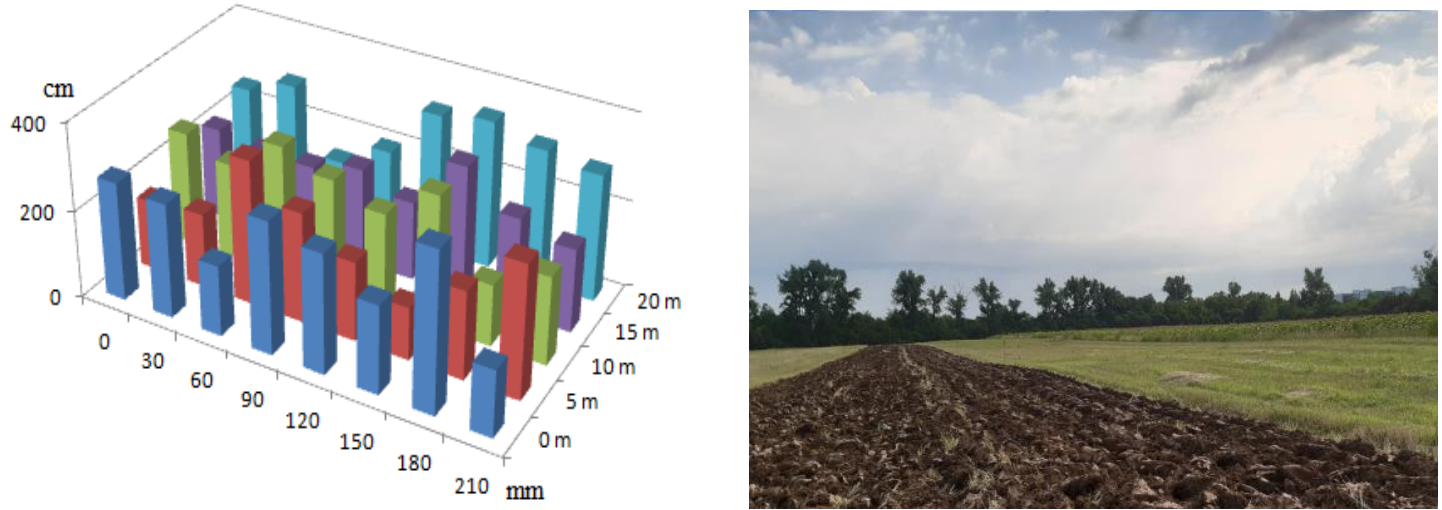

Fig. 4. Profile before passing the harrow 

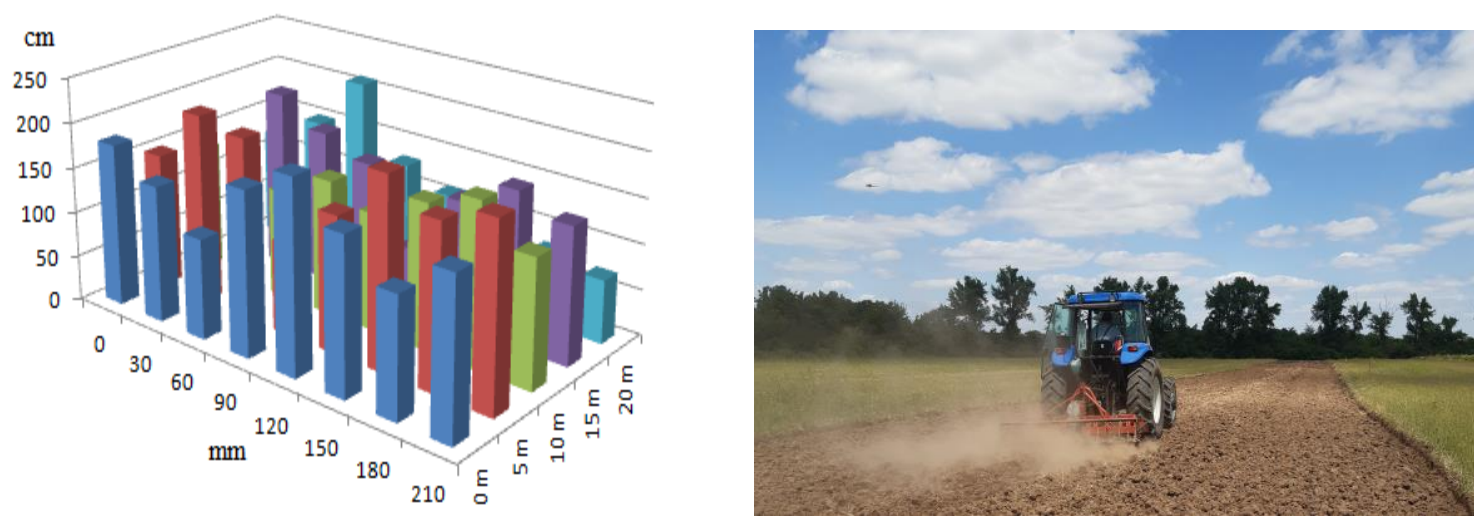

Fig. 5. Soil profile after passing the harrow

The degree of crushing of the soil processed by the harrow with rotors with vertical axis was measured according to the number of knives used for constant speed. The numerical data obtained can be put in tabular form as in Table 1.

\section{Results of measuring the degree of crushing according to the number of knives for each rotor}

\begin{tabular}{|c|c|c|c|}
\hline \multirow{2}{*}{ Intervals, mm } & \multicolumn{3}{|c|}{ Number of knives } \\
\cline { 2 - 4 } & $\mathbf{2}$ & $\mathbf{3}$ & $\mathbf{4}$ \\
\hline$<20$ & 36.8 & 46 & 55.3 \\
\hline $20-50$ & 37.7 & 33.5 & 27.7 \\
\hline $50-100$ & 18.8 & 14.7 & 12.7 \\
\hline$>100$ & 6.7 & 5.8 & 4.3 \\
\hline
\end{tabular}

Numerically it is observed that the degree of crushing is better as the number of knives on the rotor increases.

Statistical modeling of the working process of an agricultural machine deals, in particular, with those phenomena that cannot be modeled theoretically, empirical or theoretical-empirical models being necessary. The degree of soil shredding is a quality parameter of the work performed by a vertical axis rotary harrow. This parameter depends on the number of knives of each rotor, but also on the working speed and the speed of the rotors. In this study we have only data on the variation of the degree of crushing with the number of knives on the rotor.

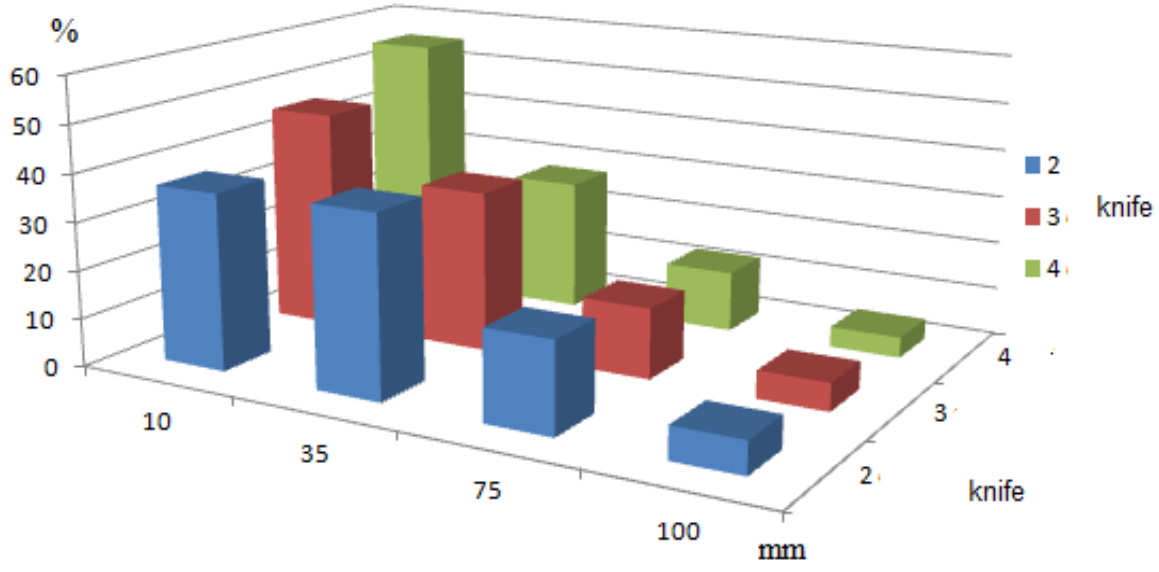

Fig. 6. Variation of the degree of crushing (as size - generalized diameter in $\mathbf{m m}$ ) with the number of knives 
Fuel consumption and theoretical productivity resulting from the experimental data lead to obtaining an economic-energy function, the specific consumption of the productivity unit, which in relation to the working speed has a representation given in Fig. 7.

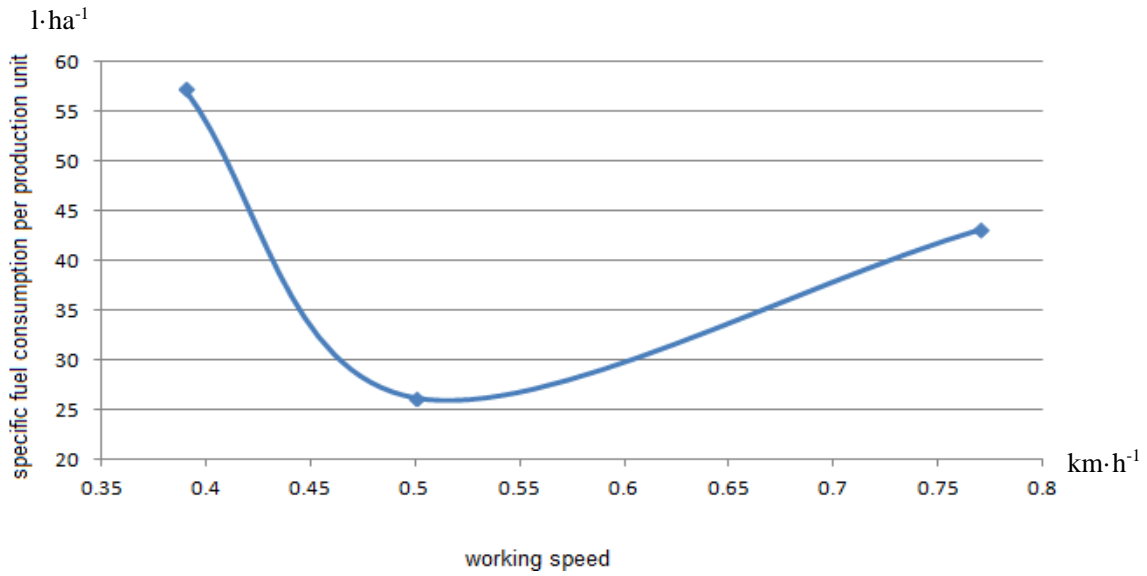

Fig. 7. Variation of consumption specific to the productivity unit with working speed

The consumption-specific function of the productivity unit provides in this variant of the experimental data, a minimum point, which, intuitively, to the mind and coordinates of such a point decreases the fuel consumption and increases the productivity. Because the importance of the existence and the values of the coordinates of the minimum point are very high, it must be retested with values of speed very close to the minimum point found. An elementary interpolation formula of the second degree for the specific consumption function is the following:

$$
C(v)=30.662-79.796 v+93.744 v^{2}, \text { in } 1 \cdot \mathrm{ha}^{-1}, v \in[0.39 ; 0.77] \mathrm{km} \cdot \mathrm{h}^{-1}
$$

with the coordinates of the minimum point:

$$
v_{o p t}=0.42 \mathrm{~km} \cdot \mathrm{h}^{-1}, C_{o p t}=36.521 \cdot \mathrm{ha}^{-1} .
$$

For the specific consumption of the productivity unit, a second degree dependence is also obtained:

$$
C_{s p}(v)=444.307-1009 v+907.596 v^{2}, \text { in } 1 \cdot\left(\mathrm{ha} \cdot \mathrm{h}^{-1}\right)^{-1}, v \in[0.39 ; 0.77] \mathrm{km} \cdot \mathrm{h}^{-1}
$$

with the coordinates of the minimum point:

$$
v_{\text {opt }}=0.556 \mathrm{~km} \cdot \mathrm{h}^{-1}, C_{\text {spopt }}=163.874 \mathrm{l} \cdot \mathrm{h} \cdot \mathrm{ha}^{-2} .
$$

$\begin{array}{ll}\text { Population size: } & 15 \\ \text { Median: } & 6990 \\ \text { Minimum: } & 2802 \\ \text { Maximum: } & 13366 \\ \text { First quartile: } & 6434 \\ \text { Third quartile: } & 8952 \\ \text { Interquartile Range: } & 2518 \\ \text { Outlier: } & 13366\end{array}$

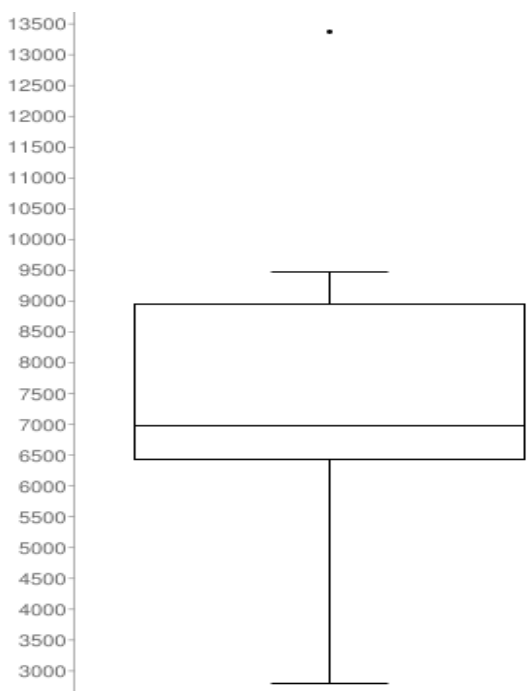

Fig. 8. Values of statistical estimators of distribution of traction force values after gross calculation in experimental records

Fig. 9. Boxplot representation of distribution of traction force values after the gross calculation from experimental records 


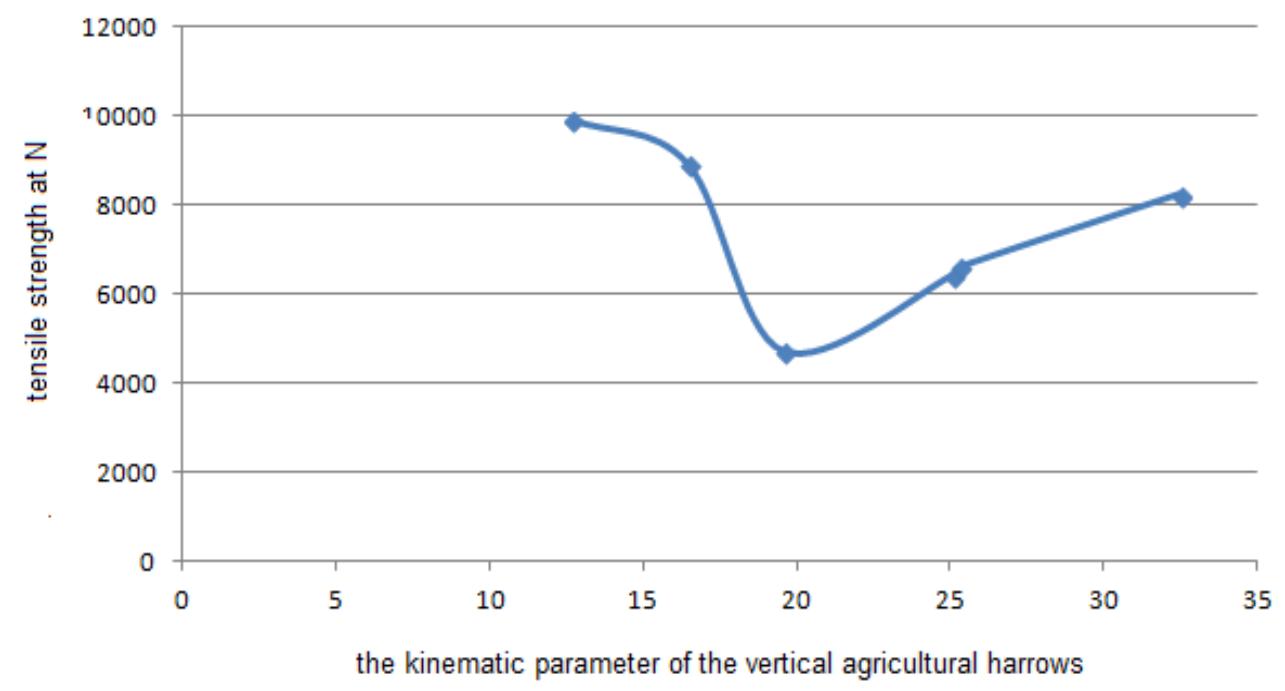

Fig. 10. Variation of tensile strength depending on the kinematic parameter of the harrow

Statistical analysis of experimental data shows that their distribution is acceptable for statistical modeling, there is only one aberrant value, Fig. 8, 9. During the experiments the working speed was timed and the rotor speed was known, so that the curve, which shows that it is possible to have an optimal point of the working process for a value of the kinematic parameter between 19 and 20, the value for which the tensile strength would fall below $4500 \mathrm{~N}$. For the tensile strength were also achieved more complex graphical representations of two variables, the kinematic parameter and the number of knives on the rotor. Second and third degree interpolation surfaces show that optimal points are possible for the work process. For example, the third degree interpolation surface (cubic) would express:

$$
\begin{aligned}
& R(\lambda, n c)=37411.718-3998.755 \lambda+9427.139 n c+1044.651 \lambda \cdot n c+ \\
& +77.1858 \lambda^{2}-9029.562 n c^{2}+6.748 \lambda^{2} \cdot n c-216.204 \lambda \cdot n c^{2}-0.979 \lambda^{3}+ \\
& +1696.955 n c^{3}
\end{aligned}
$$

where $R$-tensile strength, and $\mathrm{n}$ is the number of knives on the rotor.

\section{Conclusions}

1. There are a series of results of elementary statistical processing that signal the possible existence of optimal working points: fuel consumption per ha depending on the working speed, specific fuel consumption per unit of productivity depending on the working speed, resistance force at traction depending on the kinematic parameter, functions that give the degree of crushing and the degree of leveling depending on various adjustable parameters. These results must be studied at a higher resolution of the optimization parameters and as functions of as many variables as possible.

2. The most influential parameters of the process are the working speed, the speed of the rotors and the number of knives that equip each rotor. These parameters are all controled and scheduling, their adjustment can improve the operation of the work process.

3. There are a number of important parameters of the process, such as the specific resistance of the soil to deformation, or the coefficient of resistance to deformation depending on the speed of the working member, also the soil moisture and friction coefficients, soil texture and structure, etc.

4. The degree of the level depends intensely on the rotor speed, working speed and the number of knives (working organs). Also, due to the limited number of experience, only the dependence of the degree of the level on the work speed was studied.

5. Fuel consumption per hectare both theoretically and experimentally (although in the experimental results there are some contradictions) is shown to describe the increase in the working speed.

6. An important category of parameters of the working process of the vertical rotor harrow forms the soil characteristics: texture, chemical composition, humidity, density, resistance to penetration, cohesion, adhesion, etc. These parameters have a strong random character and cannot be chosen entirely by the operator. 


\section{References}

[1] Technologies for row cultures metrics. [online] [21.03.2021] Available at: https://www.scribd.com/document/229261539/Brochure-Clemens-Technology-for-Row-CulturesMetric

[2] Rotary tillers. [online] [21.03.2021] Available at: http://www.breviglieri.com/en/Rotary-Tillers/

[3] Constantin V. R., Research on energy optimization of the process of preparation of the germination bed at the establishment of vegetable crops, PhD thesis, Transilvania University of Brasov, 2013. [online] [21.03.2021] Available at: https://www.maschio.co.uk/products/rotary-cultivators/

[4] Foote R. L., The Volume Swept out by a Moving Planar Region, Mathematical Magazine, Vol. 79, No. 4, 2006, pp. 289-297.

[5] Ling Z. K., Chase T. R. Generating the swept area of a body undergoing planar motion. Journal of Mechanical Design, Transactions of the ASME, 118(2), pp. 186-192. DOI: 10.1115/1.2826868, 1996.

[6] Dragos L., Principiile mecanicii mediilor continue, Editura Tehnica, Bucuresti, 1983.

[7] Guglev D., Defining the critical kinematic parameters of rotary harrow with vertical axis of rotation, AGRICULTURAL SCIENCE AND TECHNOLOGY, VOL. 3, No 3, 2011, pp. 237-239.

[8] Khan K., Moses S.C., Ali M.N, Kumar A., Effect of Rotor Speed of Vertical Axis Rotary Harrow on Mean Weight Diameter of Soil Aggregates and Fuel Consumption, Trends in Biosciences 8(11), 2015, pp. 2845-2849

[9] Makange NR, Tiwari VK, Effect of Horizontal and Vertical Axis Rotavators on Soil Physical Properties and Energy Requirement, Trends in Biosciences 8(12), 2015, pp. 3225-3234.

[10] Tarverdyan A.P., Sargsyan S.F., Altunyan A., Investigation results of kinematic and dynamic indicators of tiller withvertical rotation axis in orchards soil cultivation, Annals of Agrarian Science 15, 2017, pp. 163-168.

[11] Chan CW, Wood RK, Holmes RG, Powered Harrow Operating Parameters: Effects on Soil Physical Properties, American Society of Agricultural Engineers 0001-2351/93/3605-1279, vol 36(5) , 1993, pp. 1279-1285.

[12] Usaborisut P., Sukcharoenvipharat W., Choedkiatphon S., Tilling tests of rotary tiller and power harrow after subsoiling, Journal of the Saudi Society of Agricultural Sciences, DOI: 10.1016/j.jssas.2020.05.002

[13] Sukcharoenvipharat W., Usaborisut P., Efficiency tests of rotary tiller and power harrow, Proceedings of 99th The IRES International Conference, Hanoi, Vietnam, 25th -26th January, 2018 